OPEN ACCESS

Edited by:

Yaohua Liu,

Shanghai First People's Hospital,

China

Reviewed by:

Elizabeth Jane Hovey,

New South Wales Department of

Health, Australia

Simon Hanft,

Westchester Medical Center,

United States

*Correspondence:

Song Lin

linsong2005@126.com

tORCID:

Haihui Jiang

orcid.org/0000-0002-8114-9741

Song Lin

orcid.org/0000-0001-5721-274X

Specialty section:

This article was submitted to

Neuro-Oncology and

Neurosurgical Oncology,

a section of the journal

Frontiers in Oncology

Received: 23 November 2020

Accepted: 26 April 2021

Published: 13 May 2021

Citation:

Jiang $H$, Yu K, Cui Y, Ren X, Li M, Zhang G, Yang C, Zhao X, Zhu $Q$ and Lin S (2021) Differential Predictors and Clinical Implications Associated With Long-Term Survivors in IDH Wildtype and Mutant Glioblastoma.

Front. Oncol. 11:632663.

doi: 10.3389/fonc.2021.632663

\section{Differential Predictors and Clinical Implications Associated With Long- Term Survivors in IDH Wildtype and Mutant Glioblastoma}

Haihui Jiang ${ }^{1,2+}$, Kefu Yu ${ }^{3}$, Yong Cui ${ }^{1,2}$, Xiaohui Ren ${ }^{1,2}$, Mingxiao Li $^{1,2}$, Guobin Zhang ${ }^{1,2}$, Chuanwei Yang ${ }^{1,2}$, Xuzhe Zhao ${ }^{1,2}$, Qinghui Zhu ${ }^{1,2}$ and Song Lin ${ }^{1,2,4 *}$

${ }^{1}$ Department of Neurosurgery, Beijing Tiantan Hospital, Capital Medical University, Beijing, China, ${ }^{2}$ National Clinical Research Center for Neurological Diseases, Center of Brain Tumor, Beijing Institute for Brain Disorders and Beijing Key Laboratory of Brain Tumor, Beijing, China, ${ }^{3}$ Department of Pharmacy, Beijing Tiantan Hospital, Capital Medical University, Beijing, China,

${ }^{4}$ Beijing Neurosurgical Institute, Capital Medical University, Beijing, China

Background: Glioblastoma (GBM) is the most aggressive intracranial tumor which can be divided into two subtypes based on status of isocitrate dehydrogenase (IDH). A small fraction of patients after receiving standard treatment can be long-term survivors (LTS). This study was designed to disclose the predictors and clinical implications associated with LTS in IDH wildtype and mutant GBM.

Methods: Patients who survived beyond five years after diagnosis of GBM were defined as LTS, while those with a survival less than one year were defined as shortterm survivors (STS). A total of 211 patients with diagnosis of GBM in Beijing Tiantan Hospital from January 2007 to January 2015 were enrolled, including 44 (20.9\%) LTS and 167 (79.1\%) STS. The clinical, radiological and molecular features between groups were systematically compared.

Results: Compared with STS, LTS were a subgroup of patients with a younger age at diagnosis $(P=0.006)$, a higher KPS score $(P=0.011)$, higher rates of cystic change $(P=0.037), O^{6}$-methylguanine-DNA methyltransferase (MGMT) promoter methylation $(P=0.007)$, and IDH mutation $(P=0.049)$, and more likely to have undergone gross total resection $(P<0.001)$. Survival analysis demonstrated that $L T S$ with wildtype IDH conferred a longer progression-free survival (66.0 vs. 27.0 months, $P=0.04$ ), but a shorter postprogression survival (46.5 months vs. not reached, $P=0.0001$ ) than those of LTS with mutant IDH. LTS with mutant IDH showed a trend towards increased survival after receiving re-operation $(P=0.155)$ and reirradiation $(P=0.127)$, while this clinical benefit disappeared in the subset of LTS with wildtype IDH $(P>0.05)$.

Conclusion: The prognostic value and therapeutic implications associated with LTS in GBM population significantly differed on the basis of IDH status. Our findings provide a new approach for physicians to better understand the two subtypes of GBM, which may assist in making more tailored treatment decisions for patients.

Keywords: glioblastoma, long-term survivor, IDH, precision medicine, treatment 


\section{INTRODUCTION}

Glioblastoma (GBM) is one of the worldwide intractable malignancies in adults (1). Despite advances in the therapeutic regimens during past few decades, the clinical outcomes of patients with GBM have not been substantially improved (24). It is reported that most of patients will progress within one year after resection, and the median survival is less than two years (4). Although the survival rate of GBM remains unfavorable, there are still a few patients who demonstrate an extraordinary response to treatment with a prolonged progression-free survival (PFS); some patients even survive more than five years (5-7). But unfortunately, the intrinsic characteristics of these long-term survivors (LTS) are still unclear (5).

Previous studies have established that isocitrate dehydrogenase (IDH) mutation was a strong predictor associated with long-term survival of patients with GBM $(8,9)$, which has led the neuropathologists to reclassify GBM into two molecular subtypes: IDH-wildtype (IDH-wt) GBM and IDH-mutant (IDH-mut) GBM (10). Traditionally, IDH-mut GBM is regarded as a secondary malignancy that had transformed from a low-grade diffuse glioma (11). Meanwhile, IDH-wt GBM, which represents the major component $(>90 \%)$ of the whole GBM cohort, is clinically defined as primary GBM (10). There are some studies that have explored the predictors for becoming a long-term survivor within the GBM population $(5,6,8,9)$. But up to now, no studies have compared the characteristics and clinical implications associated with LTS between IDH-wt and IDHmut GBM.

Therefore, in the present study, we systematically compared the clinical, radiological and molecular characteristics between LTS and short-term survivors (STS) in the cohort of GBM. Furthermore, the intrinsic characteristics and clinical implications correlated with LTS between IDH-wt and IDHmut GBM have also been explored. We found that the clinical, radiological, and molecular features of IDH-wt and IDH-mut LTS were significantly different and the two subtypes of LTS showed distinct PFS and post-progression survival (PPS), which can help the physicians to better understand GBM and may contribute to making more tailored treatment decisions for patients.

\section{MATERIALS AND METHODS}

\section{Patient Cohort}

Forty-four patients who survived beyond five years after diagnosis of GBM (LTS) and 167 patients who survived less than one years after diagnosis of GBM (STS) between January 2007 and January 2015 in Beijing Tiantan Hospital affiliated to Capital Medical University were selected for inclusion in this study. Pathology review was performed by two experienced neuropathologists according to the 2016 World Health Organization (WHO) classification schema (10). All the patients, in our institution, once diagnosed with GBM are recommended to proceed with post-operative combination radiation and chemotherapy. However, there are still a small number of patients who missed out on chemotherapy or radiation for personal reasons. Radiation was performed within one month after operation, with a total dose of $60 \mathrm{~Gy}$ which was further divided into 30 fractions. The adjuvant chemotherapy regimen was mainly nimostine (ACNU) and temozolomide (TMZ), according to the previously described protocol (12). When tumor progressed, re-operation and reirradiation were performed if possible. Rechallenge with chemotherapy which commonly consisted of a combination of bevacizumab and temozolomide (TMZ) could be also attempted if patients showed relatively normal laboratory tests and maintained a reasonable performance status (ECOG: 0-2) (13).

\section{Radiological Evaluation}

The radiological evaluation was performed by 3 experienced neuroradiologists who were blind to the clinical outcome of patients. Radiological features included tumor location, tumor size, enhancement, cystic change, and extent of resection. The calculation of tumor size was mainly based on the T1-weighted imaging (T1WI) contrast enhanced area. Enhancement was classified into three subtypes: solid, ring, irregular (Figure 1) (14). Solid subtype was relatively uniformly enhanced and concurrent with a well-circumscribed edge. Ring subtype was characterized by a ring-like enhancement with central necrosis or cyst. Irregular subtype had scattered enhancement which was irregularly shaped. A cystic tumor was defined as those with a large cyst cavity comprising at least half of the whole tumor and the cyst was filled with fluid that showed a radiographic appearance similar to cerebrospinal fluid on T2-weighted imaging (T2WI) (Figure 1) (15). Hence, tumors with fluidfilled cysts and those with large central necrosis were both regarded as cystic tumors in this study. Within 72 hours after operation, an enhanced magnetic resonance imaging (MRI) was carried out to assess the extent of resection (EOR). EOR was calculated on the basis of contrast-enhanced T1WI, according to the following equation: (preoperative tumor volume postoperative tumor volume)/preoperative tumor volume.

\section{Molecular Biomarkers Detection}

Abnormality of chromosome $1 \mathrm{p} / 19 \mathrm{q}$, IDH mutation and $\mathrm{O}^{6}$ methylguanine-DNA methyltransferase (MGMT) promoter methylation were respectively analyzed by fluorescence in situ hybridization (FISH) (16), Sanger sequencing (17) and pyrosequencing (18), according to previously described methods (Figure 2). Ki-67 index was detected by immunohistochemistry (IHC) staining which was done with a monoclonal mouse antibody (1:80 dilution, Dako) (Figure 2). The expression level of the Ki-67 index was defined as either high $(\geq 30 \%)$ or low $(<30 \%)$ for interpretation, according to the percentage of IHCpositive cells (19).

\section{Follow-Up}

After operation, patients were regularly followed up with brain MRI scans until death. MRIs were performed at an interval of three months, or more frequent in the event of clinical changes, 


\section{I: enhancement}

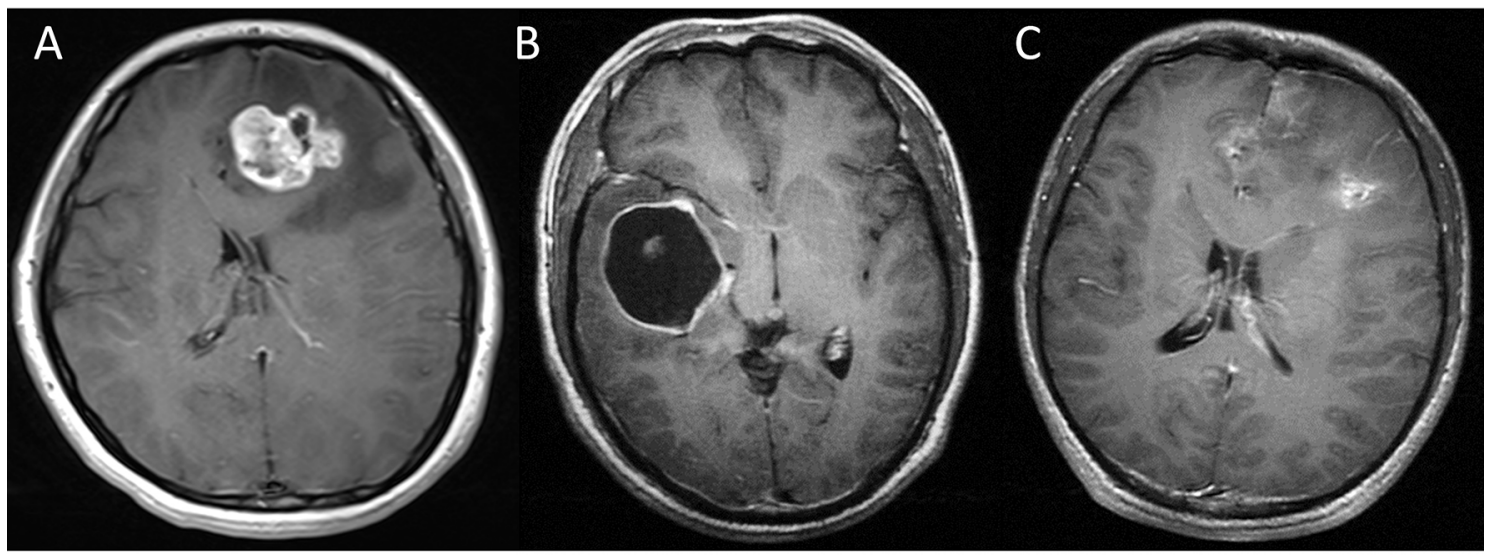

\section{II: cystic}

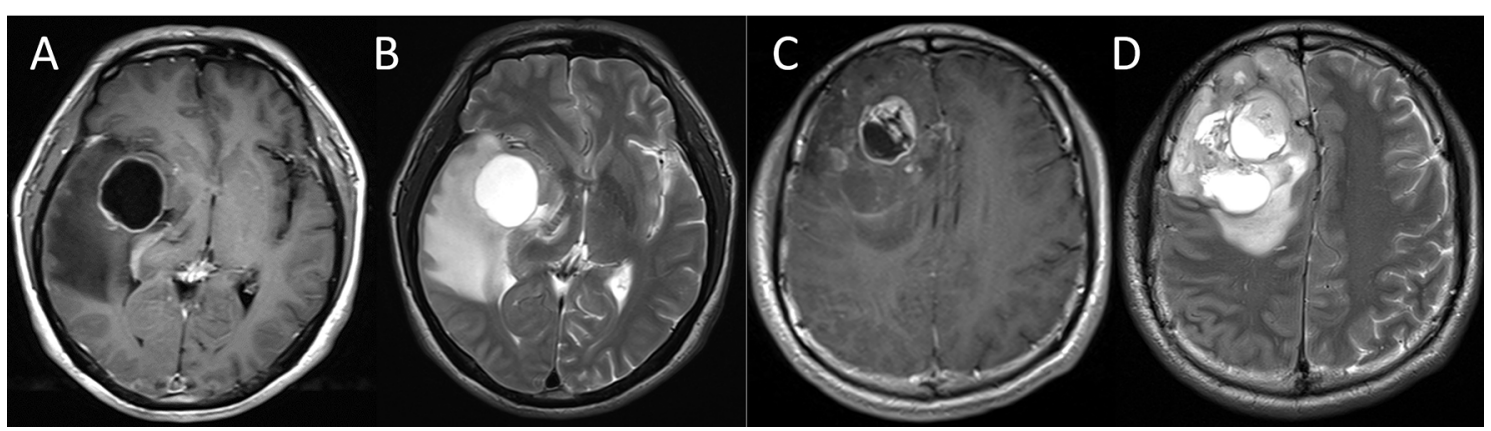

FIGURE 1 | Panel I: Representative images of solid (A), ring (B), and irregular (C) enhancement. Panel II: Typical images of cystic GBM. A tumor in the right insular lobe with fluid-filled cysts (A, B) and a tumor in the right frontal lobe with large central necrosis (C, D).

such as seizures or neurologic deterioration. Progression pattern was divided into local and non-local subtypes based on the preoperative and serial postoperative radiographic images (Figure S1). Local recurrence was those with lesion located in the resection cavity or in continuity with it, or less than $2 \mathrm{~cm}$ from the primary tumor margins, while the recurrence lesion border beyond $2 \mathrm{~cm}$ from the original cavity was defined as nonlocal failure (20). PFS was defined as the time period from the date of operation to the date of progression or recurrence demonstrated by MRI, death or last follow up. Overall survival (OS) was defined as the time period from the date of initial operation to the date of death or last follow-up. Timespan between the first progression and death/last follow-up was defined as post-progression survival (PPS). The median followup of this cohort was 71.5 (range: 1.0-130.0) months. There were $195(92.4 \%)$ patients with progression and 176 (83.4\%) patients had died by the time of data analysis.

\section{Statistical Analysis}

All the analyses were performed with SPSS (version 22.0, Chicago, IL, USA) and R software (http://www.r-project.org, The $\mathrm{R}$ Foundation). Comparisons of categorical variables between the groups were performed using chi-square test or Fisher's exact test, while differences in age at diagnosis, tumor size and Karnofsky performance scale (KPS) score were evaluated by student $\mathrm{t}$-test. The variables with $\mathrm{P}$ values less than 0.05 were entered into the multivariate logistic regression analysis to identify the independent predictors of LTS. Survival rates were calculated using the Kaplan-Meier methods, and differences were compared by log-rank tests. All tests were two-sided, and difference with a $P$ value less than 0.05 was considered to be statistically significant.

\section{RESULTS}

\section{Patient Population}

We identified 211 patients, including 44 (20.9\%) LTS and 167 (79.1\%) STS. Of these 44 LTS, there were 17 (38.6\%) patients with wildtype IDH and $27(61.4 \%)$ patients with mutant IDH. Our cohort consisted of 126 males and 85 females with a mean age of $49.0 \pm 11.8$ years. All the clinical, radiological, and molecular characteristics of patients were summarized in Table 1. 

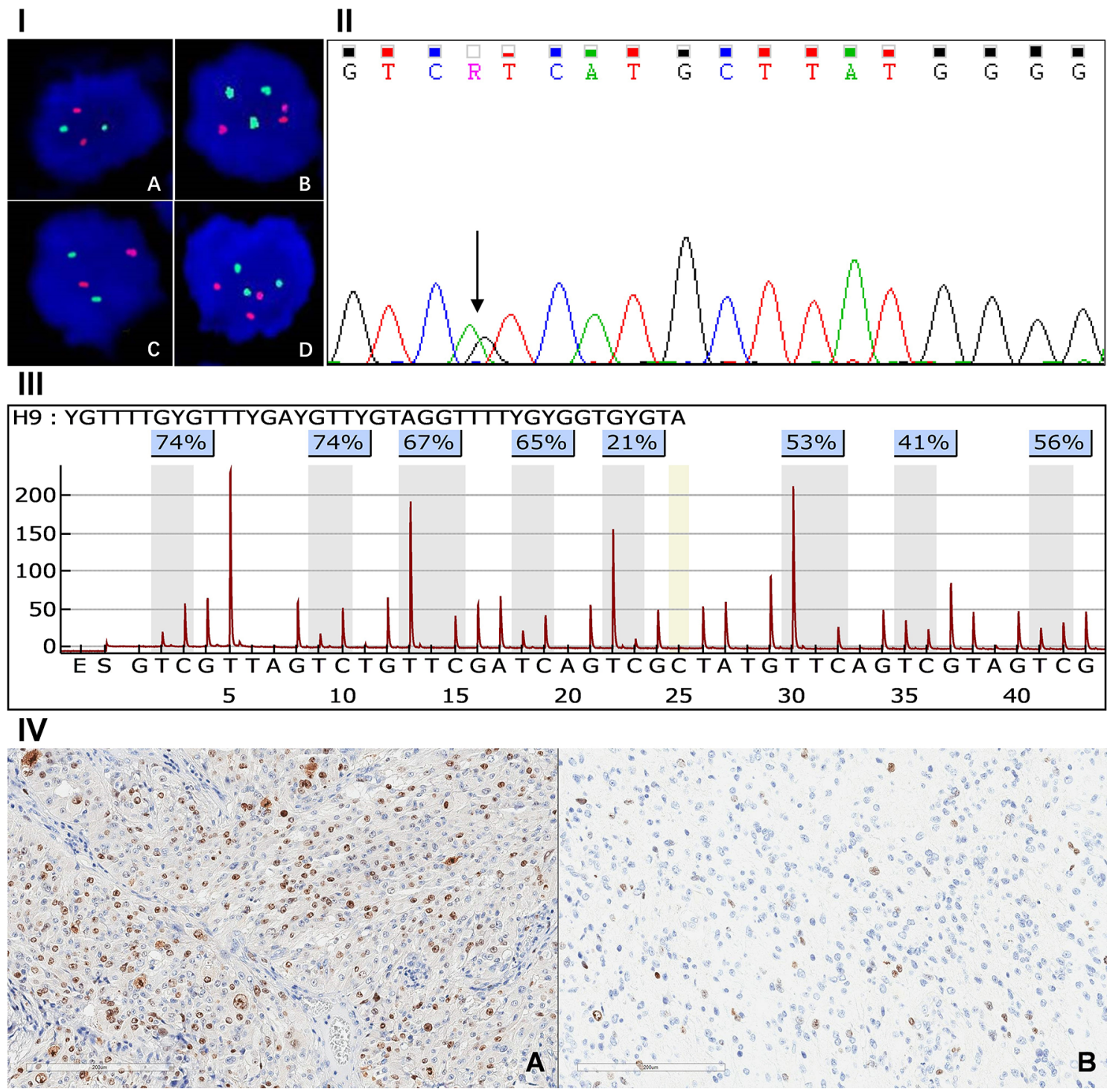

FIGURE 2 | Panel I, FISH detection result of 1q/19p polysomy: 1p intact (A), 1q polysomy (B), 19q intact (C), 19p polysomy (D). Panel II: Sanger sequence of IDH1 mutation. Panel III: Assay of MGMT promoter methylation in GBM. Panel IV: Immunohistochemical staining of high (A) and low (B) Ki-67 index.

\section{Comparison of Baseline Characteristics Between STS and LTS}

LTS patients were younger $(41.2 \pm 11.1$ vs. $49.9 \pm 11.3, P<0.001)$ and had higher KPS score $(82.2 \pm 8.3$ vs. $76.2 \pm 14.9, P=0.002)$ than the STS. But they shared a similar gender ratio and tumor size $(P>0.05)$. There were more frontal tumors in LTS $(59.1 \% v s$. $35.9 \%, P=0.039)$, but no significant difference has been observed in the laterality $(P=0.138)$. The enhancement features were also similar between STS and LTS $(P=0.139)$, while LTS showed a higher frequency of cystic change $(56.8 \%$ vs. $19.2 \%, P<0.001)$. With respect to the treatment information, LTS patients were more likely to have undergone gross-total resection (GTR) (86.4\% vs. $31.7 \%, P<0.001)$, and to have received chemotherapy $(100.0 \%$ vs. $91.6 \%, P=0.084)$ and radiotherapy (100.0\% vs. $86.2 \%, P=0.005)$, compared with the STS. The molecular profile of LTS patients was characterized by a higher rate of MGMT promoter methylation (70.6\% vs. $34.9 \%$, $P<0.001)$, IDH mutation (61.4\% vs. $13.2 \%, P<0.001)$, and $1 \mathrm{q} /$ 19 p co-polysomy $(25.7 \%$ vs. $12.6 \%, P=0.047)$. The Ki-67 index was similar among groups $(P=0.510)$ (Table 1).

On the basis of these predictors identified by univariate analyses, a multivariate logistic regression model was built. The final results showed that age $<50$ years (odds ratio $[\mathrm{OR}]=1.081,95 \%$ confidence interval [CI]: 1.022-1.141, $P=0.006)$, KPS score $\geq 70$ $(\mathrm{OR}=22.354,95 \% \mathrm{CI}: 2.028-246.449, P=0.011)$, cystic change (OR = 3.791, 95\% CI: 1.082-13.275, $P=0.037)$, GTR (OR = 18.731, 95\% CI: 4.636-75.690, $P<0.001)$, MGMT promoter methylation 
TABLE 1 | Comparisons of baseline characteristics between short- and longterm survivors.

\begin{tabular}{|c|c|c|c|c|}
\hline Variables & All $(n=211)$ & STS $(n=167)$ & LTS ( $n=44)$ & $P$ value \\
\hline Age at diagnosis (years) & $49.0 \pm 11.8$ & $49.9 \pm 11.3$ & $41.2 \pm 11.1$ & $<0.001$ \\
\hline Gender & & & & 0.432 \\
\hline Male & $126 / 211$ & 102/167 & $24 / 44$ & \\
\hline KPS score & $80.0 \pm 14.2$ & $76.2 \pm 14.9$ & $82.2 \pm 8.3$ & 0.002 \\
\hline Tumor size (mm) & $49.2 \pm 18.9$ & $49.5 \pm 19.1$ & $48.1 \pm 18.3$ & 0.652 \\
\hline Tumor location & & & & $0.039^{*}$ \\
\hline Frontal & $86 / 211$ & $60 / 167$ & $26 / 44$ & \\
\hline Temporal & $56 / 211$ & $45 / 167$ & $11 / 44$ & \\
\hline Parietal & $35 / 211$ & $32 / 167$ & $3 / 44$ & \\
\hline Occipital & $18 / 211$ & $16 / 167$ & $2 / 44$ & \\
\hline Others & $16 / 211$ & $14 / 167$ & $2 / 44$ & \\
\hline Laterality & & & & 0.138 \\
\hline Right & $92 / 211$ & $67 / 167$ & $25 / 44$ & \\
\hline Left & $95 / 211$ & $80 / 167$ & $15 / 44$ & \\
\hline Bilateral & $24 / 211$ & $20 / 167$ & $4 / 44$ & \\
\hline Enhancement & & & & 0.139 \\
\hline Solid & $144 / 211$ & $118 / 167$ & $26 / 44$ & \\
\hline Ring & $38 / 211$ & $30 / 167$ & $8 / 44$ & \\
\hline Irregular & $29 / 211$ & $19 / 167$ & $10 / 44$ & \\
\hline Cystic change & & & & $<0.001$ \\
\hline Yes & $57 / 211$ & $32 / 167$ & $25 / 44$ & \\
\hline Extent of resection & & & & $<0.001$ \\
\hline GTR & $91 / 211$ & $53 / 167$ & $38 / 44$ & \\
\hline Chemotherapy & & & & 0.084 \\
\hline Temozolomide & $170 / 211$ & $134 / 167$ & $36 / 44$ & \\
\hline Nimostine & $27 / 211$ & 19/167 & $8 / 44$ & \\
\hline None & $14 / 211$ & $14 / 167$ & $0 / 44$ & \\
\hline Radiotherapy & & & & $0.005^{\star}$ \\
\hline Yes & $188 / 211$ & $144 / 167$ & $44 / 44$ & \\
\hline Recurrence pattern & & & & 0.838 \\
\hline Local & $156 / 195$ & $134 / 167$ & $22 / 28$ & \\
\hline MGMT promotor & & & & $<0.001$ \\
\hline Methylation & $68 / 160$ & $44 / 126$ & $24 / 34$ & \\
\hline $\mathrm{IDH}$ & & & & $<0.001$ \\
\hline Mutation & $55 / 211$ & $28 / 167$ & $27 / 44$ & \\
\hline 1q/19p co-polysomy & & & & 0.047 \\
\hline Yes & $30 / 202$ & $21 / 167$ & $9 / 35$ & \\
\hline Ki-67 index & & & & 0.510 \\
\hline High & $70 / 193$ & $59 / 158$ & $11 / 35$ & \\
\hline
\end{tabular}

KPS, Karnofsky performance scale; GTR, gross-total resection; MGMT, $O^{6}$ methylguanine-DNA-methyltransferase; IDH, isocitrate dehydrogenase.

*by Fisher exact test.

(OR $=5.553,95 \%$ CI: $1.591-19.379, P=0.007)$, and IDH mutation $(\mathrm{OR}=4.321,95 \% \mathrm{CI}: 1.007-18.535, P=0.049)$ were confirmed as predictive factors for LTS (Table 2).

\section{Comparison of Baseline Characteristics Between IDH-Wt and IDH-Mut LTS}

Compared with the IDH-wt LTS, IDH-mut LTS had a lower rate of solid enhancement ( $44.4 \%$ vs. $82.3 \%, P=0.036)$, but higher rates of cystic change $(70.4 \%$ vs. $35.3 \%, P=0.022)$, local recurrence $(95.0 \%$ vs. $37.5 \%, P=0.003)$, and $1 \mathrm{q} / 19 \mathrm{p}$ copolysomy $(44.4 \%$ vs. $5.9 \%, P=0.018)$. The Ki-67 index of IDH-mut LTS was lower than that in the IDH-wt LTS, but the difference was not statistically significant $(16.7 \%$ vs. $47.1 \%, P=$ 0.053) (Table 3).
TABLE 2 | Results of multivariate logistic regression analysis.

\begin{tabular}{|c|c|c|c|}
\hline Variables & Odds ratio (OR) & 95\% Confidence interval (Cl) & $P$ value \\
\hline \multicolumn{4}{|c|}{ Age at diagnosis } \\
\hline$<50$ years & 1.081 & $1.022-1.141$ & 0.006 \\
\hline \multicolumn{4}{|l|}{ KPS score } \\
\hline$\geq 70$ & 22.354 & 2.028-246.449 & 0.011 \\
\hline \multicolumn{4}{|l|}{ Cystic change } \\
\hline Yes & 3.791 & $1.082-13.275$ & 0.037 \\
\hline \multicolumn{4}{|l|}{ Extent of } \\
\hline \multicolumn{4}{|l|}{ resection } \\
\hline GTR & 18.731 & $4.636-75.690$ & $<0.001$ \\
\hline \multicolumn{4}{|c|}{ MGMT promotor } \\
\hline Methylation & 5.553 & $1.591-19.379$ & 0.007 \\
\hline \multicolumn{4}{|l|}{$\mathrm{IDH}$} \\
\hline Mutation & 4.321 & $1.007-18.535$ & 0.049 \\
\hline
\end{tabular}

TABLE 3 | Comparisons of baseline characteristics between IDH-wt and IDHmut long-term survivors.

\begin{tabular}{|c|c|c|c|}
\hline Variables & IDH-wt (n=17) & IDH-mut (n=27) & $P$ value \\
\hline Age at diagnosis (years) & $41.1 \pm 12.3$ & $41.3 \pm 10.5$ & 0.938 \\
\hline Gender & & & 0.651 \\
\hline Male & $10 / 17$ & $14 / 27$ & \\
\hline KPS score & $80.0 \pm 8.7$ & $84.7 \pm 7.4$ & 0.115 \\
\hline Tumor size (mm) & $52.4 \pm 16.3$ & $45.3 \pm 19.2$ & 0.213 \\
\hline Tumor location & & & 0.351 \\
\hline Frontal & $10 / 17$ & $16 / 27$ & \\
\hline Temporal & $6 / 17$ & $5 / 27$ & \\
\hline Parietal & $0 / 17$ & $3 / 17$ & \\
\hline Occipital & $1 / 17$ & $1 / 27$ & \\
\hline Others & $0 / 17$ & $2 / 27$ & \\
\hline Laterality & & & 0.342 \\
\hline Right & $8 / 17$ & $17 / 27$ & \\
\hline Left & $8 / 17$ & $7 / 27$ & \\
\hline Bilateral & $1 / 17$ & $3 / 27$ & \\
\hline Enhancement & & & 0.036 \\
\hline Solid & $14 / 17$ & $12 / 27$ & \\
\hline Ring & $2 / 17$ & $6 / 27$ & \\
\hline Irregular & $1 / 17$ & $9 / 27$ & \\
\hline Cystic change & & & 0.022 \\
\hline Yes & $6 / 17$ & $19 / 27$ & \\
\hline Extent of resection & & & $0.380^{*}$ \\
\hline GTR & $16 / 17$ & $22 / 27$ & \\
\hline Chemotherapy & & & $0.125^{\star}$ \\
\hline Temozolomide & $16 / 17$ & $20 / 27$ & \\
\hline Nimostine & $1 / 17$ & $7 / 27$ & \\
\hline Radiotherapy & & & NA \\
\hline Yes & $17 / 17$ & $27 / 27$ & \\
\hline Recurrence pattern & & & $0.003^{\star}$ \\
\hline Local & $3 / 8$ & $19 / 20$ & \\
\hline MGMT promotor & & & 1.0 \\
\hline Methylation & $12 / 17$ & $12 / 17$ & \\
\hline 1q/19p co-polysomy & & & $0.018^{\star}$ \\
\hline Yes & $1 / 17$ & $8 / 18$ & \\
\hline $\mathrm{Ki}-67$ index & & & 0.053 \\
\hline High & $8 / 17$ & $3 / 18$ & \\
\hline
\end{tabular}

IDH, isocitrate dehydrogenase; KPS, Karnofsky performance scale; GTR, gross-total resection; MGMT, $\mathrm{O}^{6}$-methylguanine-DNA-methyltransferase; NA, not applicable. *by Fisher exact test. 


\section{Differential Clinical Implications Between IDH-wt and IDH-Mut LTS}

With respect to the prognostic implication of IDH in LTS patients, the survival rates between IDH-wt and IDH-mut subgroups in terms of PFS, OS, and PPS were compared. Results demonstrated that IDH-wt LTS shared a similar survival rate with IDH-mut LTS in regard to OS $(P=0.262)$

(Figure 3A). But interestingly, the median PFS of IDH-wt LTS was unexpectedly longer than that of IDH-mut LTS (66.0 vs. 27.0 months, $P=0.040)$. Conversely, the median PPS of IDH-wt LTS was significantly shorter than that of IDH-mut LTS (46.5 months vs. not reached, $P=0.0001$ ) (Figures 3B, C).

Considering the distinct survival distribution in PFS and PPS between IDH-wt and IDH-mut LTS, we explored the clinical implication of recurrence pattern in LTS patients. Of these 44 LTS, 22 patients (including 3 IDH-wt LTS and 19 IDH-mut LTS) experienced local recurrence at a median period of 18.5 (10.029.0) months and 6 patients (including 5 IDH-wt LTS and 1 IDH-mut LTS) experienced non-local recurrence at a median period of 45.0 (16.2-76.8) months, which imparted a significant difference $(P=0.043)$ (Figure 4A). Moreover, the percent of death was also markedly different between patients with local and non-local recurrence $(P=0.006)$ (Figure 4B).

Of the 28 patients who underwent tumor progression, 12 (42.9\%) patients (including 4 IDH-wt LTS and 8 IDH-mut LTS) received re-operation, 15 (53.6\%) patients (including $5 \mathrm{IDH}-\mathrm{wt}$ LTS and 10 IDH-mut LTS) received reirradiation, and all (100.0\%) patients (including 8 IDH-wt LTS and 20 IDH-mut LTS) received rechallenge with chemotherapy (Figure S2). Kaplan-Meier plots demonstrated that IDH-mut LTS showed a trend towards increased survival after receiving re-operation $(P=$ $0.155)$ and reirradiation $(P=0.127)$, while this clinical benefit disappeared in the subset of IDH-wt $(P>0.05)$ (Figure 5).

\section{DISCUSSION}

GBM is the most aggressive intracranial malignancy, with rapid growth, inevitable recurrence and high mortality (4). Only a small fraction of patients can achieve a long-term survival after surgical resection and chemoradiotherapy. But the diagnostic threshold of LTS varies significantly in the pre-existing literatures, ranging from 2 years to 5 years $(5,9,18,21)$. As the median survival of patients with GBM is about 1 year and 5 years survival rate is regarded as a predictor of better tumor control, patients who survived beyond 5 years after diagnosis, in this study, were identified as LTS, while those with a survival less than 1 year were defined as STS (5). We systematically compared the clinical, radiological, and molecular features between STS and LTS. Although the characteristics of LTS in GBM have been
A
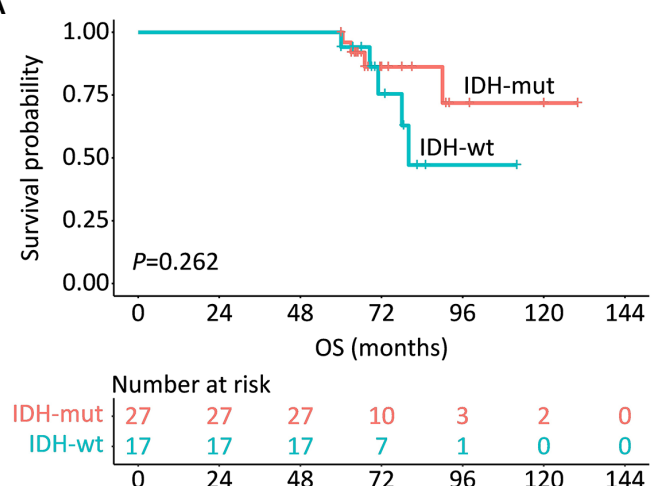

C

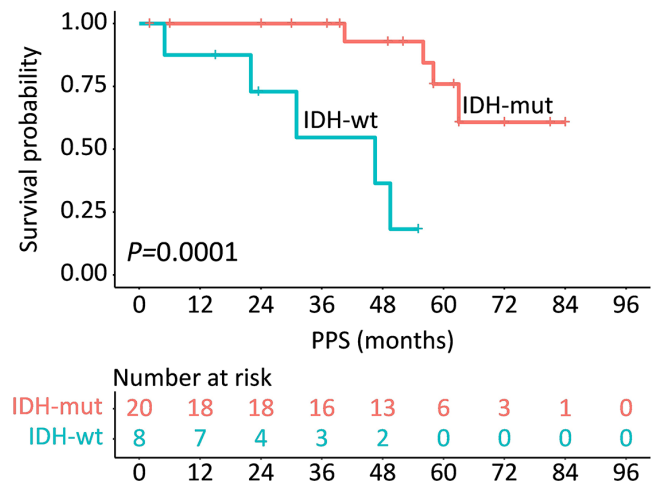

B
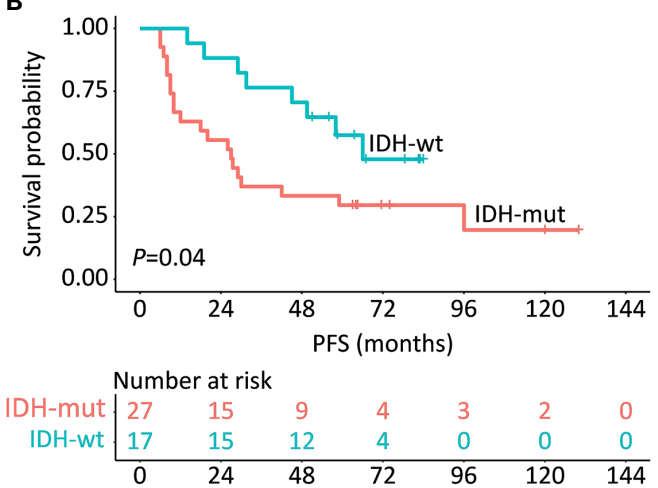

FIGURE 3 | Survival comparisons in regards to OS (A), PFS (B) and PPS (C) between IDH-wt and IDH-mut LTS. 


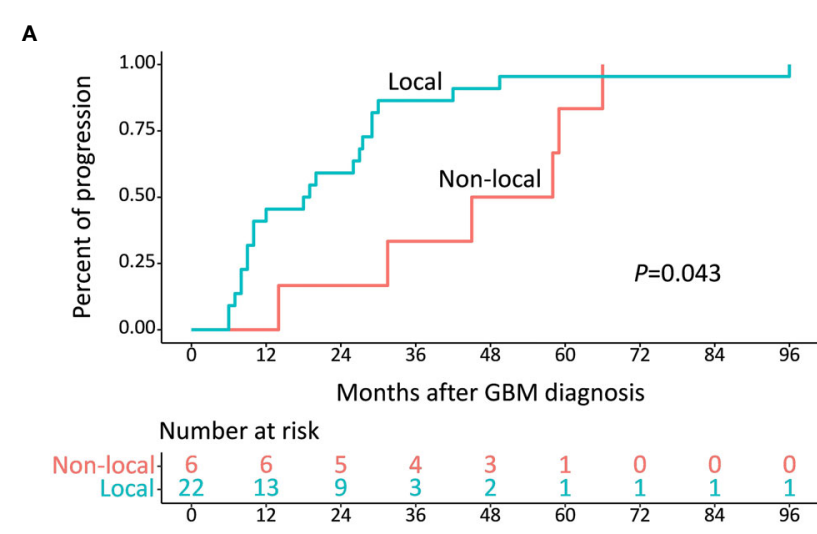

B

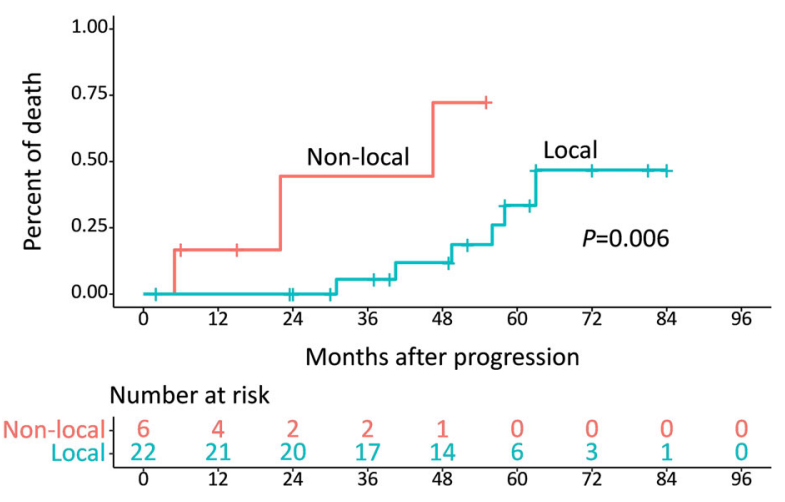

FIGURE 4 | (A) Median time from diagnosis to development of progression was 18.5 months for local recurrence and 45.0 months for non-local recurrence $(P=0.043)$. (B) Median survival after diagnosis of non-local recurrence was 46.5 months, which was shorter than that of local recurrence $(P=0.006)$

widely investigated $(5,6,8,9,18,21,22)$, there is no study devoted to exploring the intrinsic properties of IDH-wt and IDH-mut LTS. To our knowledge, it's the first study that was aimed to disclose the differential predictors and clinical implications between IDH-wt and IDH-mut LTS. Our results demonstrated that IDH-mut LTS had a lower rate of solid enhancement, but higher rates of cystic change, 1q/19p copolysomy, and local recurrence. IDH-mut LTS showed a shorter PFS, but a significantly prolonged PPS than those of IDH-wt LTS.

Younger age, better performance status, and higher resection degree are universally regarded as predictors of superior survival in GBM $(5,23-27)$. In this study, we found that LTS were a subgroup of patients who had a younger age, a higher KPS score, and a more radical resection. In 2014, Field et al. compared the characteristics between patients who survived more than 2 years and those with a survival less than 6 months (23). Final results showed that younger age, better performance status, gross macroscopic resection, and clinical trial participation were independent predictors of LTS (23). Similarly, some authors have explored the features of LTS who were defined as surviving over 3 years by comparing with controls and found that LTS were younger, had better performance status, and were more likely to have received GTR and adjuvant chemotherapy $(24,25)$. These studies, however, enrolled a small number of LTS, which to a certain extent decreased the reliability of conclusions. Recently, a report of 2249 LTS from National Cancer Database maintained that factors associated with improved 5-year survival were younger age, female gender, less medical comorbidities, non-white race, higher salary, left-sided tumors and tumors outside the brainstem, and radiotherapy (5). However, the molecular parameters have not been explored in the study.

In addition to the clinical factors, the molecular biomarkers, such as IDH and MGMT, are also closely correlated with patient's survival $(18,24,28-30)$. Most of the prior studies repeatedly demonstrated that IDH mutation was a prognostic factor associated with prolonged survival $(18,31)$. GBM was divided into two major categories based on the status of IDH since 2016 when the latest World Health Organization classification schema of brain tumor was issued (10). In our study, the frequencies of IDH mutation and MGMT promoter methylation in LTS were significantly higher than those in STS, which was consistent with previous findings (18). Barbus et al. found that IDH mutation was more prevalent in LTS (28). However, in a larger cohort study of comparative patients, the presence of IDH mutation was not significantly associated with LTS (9). Of note, their further analysis showed that significantly more LTS were MGMT methylated and IDH mutant (9). MGMT is a DNA repair protein involved in reversing methylation damage from alkylating agent (32). It's universally acknowledged that methylated MGMT is linked to increased chemosensitivity and generally confers an improved survival (32). The frequency of MGMT promoter methylation of LTS was $70.6 \%$ in our study, which was in accordance with prior results $(6,9,24,29,30)$. It is well-established that methylated MGMT is more prevalent in LTS of GBM, compared with the STS patients $(18,24,29,30)$. Together these suggest that MGMT methylation is one of the most important features of LTS.

Within the group of LTS, solid enhancement seemed to be more likely occurred in IDH-wt LTS, while cystic change was predominant in IDH-mut group. Rathore and colleagues divided GBM into three distinct subtypes based on the signature of enhancement and found that classical tumors were more prevalent in the solid subtype which showed the worst clinical outcome (14). Cystic change is confirmed as a prognostic factor associated with favorable outcomes $(33,34)$. Some authors held that cystic GBM may develop from malignant transformation of a previously undiagnosed cystic low-grade glioma (34). This explained why cystic change occurred more frequently in IDHmut GBM cases which had a history of low-grade glioma within our cohort. Utsuki et al. (34) believed cystic GBM was less aggressive and had little infiltration of the peritumoral brain tissue, which is consistent with the lower Ki-67 index demonstrated in the tumors of the IDH-mut GBM group in our study. Furthermore, we found IDH-mut LTS presented with a higher rate of 1q/19p co-polysomy than IDH-wt GBM. In 2017, Zeng et al. analyzed the prognostic implication of $1 q / 19 p$ 


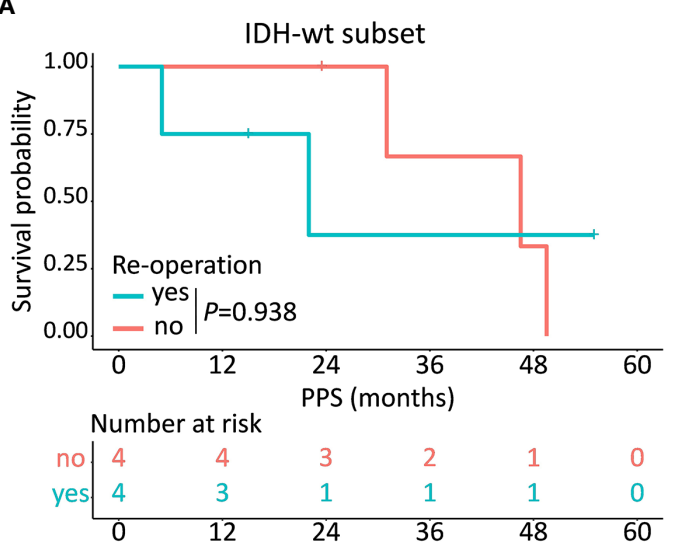

C

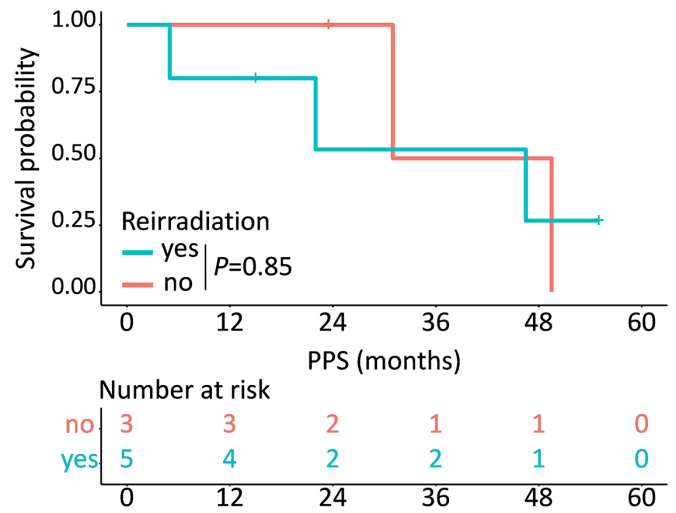

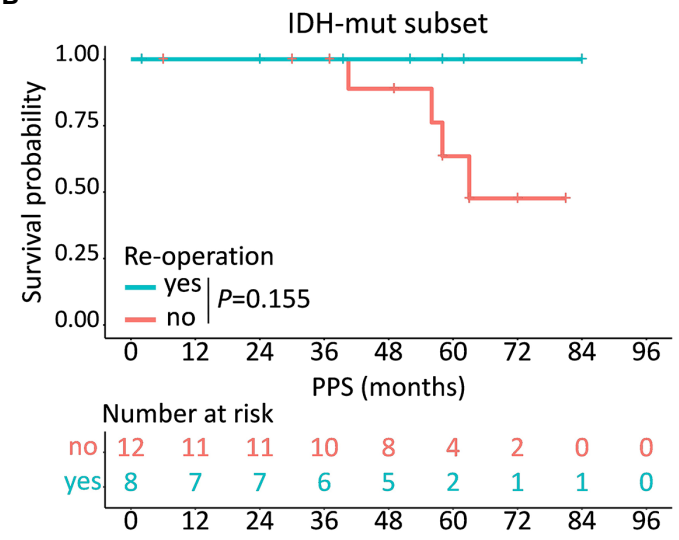

D

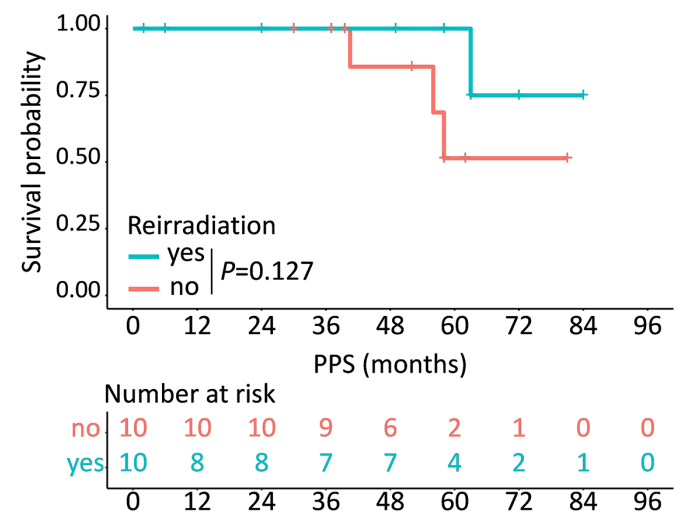

FIGURE 5 | Prognostic implications of different kinds of treatment regimens in IDH-wt and IDH-mut subtypes. In the subset of IDH-wt LTS, no obvious clinical benefit has been observed after receiving re-operation $\mathbf{( A )}$ or reirradiation $\mathbf{( C )}$ while IDH-mut LTS show a trend towards increased survival after receiving re-operation (B) and reirradiation (D).

polysomy in two large cohorts of astrocytic gliomas and found co-polysomy was an independent prognostic factor associated with prolonged survival (35). All the findings imply that IDHmut GBM seems to be a less aggressive brain tumor, compared with IDH-wt GBM.

The most interesting finding of our study was that IDH-wt LTS showed a significantly higher rate of non-local failure compared with that in IDH-mut group, which determined the different survival distribution spectrum between IDH-wt and IDH-mut LTS. As we all know, non-local failure is a fatal event which commonly occurs later than local failure (20). In our study, the median time period from diagnosis to local failure was 18.5 months, which was shorter than the interval between diagnosis and non-local failure. Meanwhile, IDH-wt LTS had a higher rate of non-local failure than that of IDH-mut LTS. Therefore, the favorable PFS among IDH-wt LTS could be ascribed to a higher rate of non-local failure. Notably, although IDH-wt LTS conferred a longer PFS, the PPS of these patients was significantly shorter than IDH-mut LTS. With an attempt to interpret the opposite result observed in PPS, we explored the relationship between postprogression treatments and PPS. Finally, the survival analyses demonstrated that IDH-mut LTS showed a trend towards increased survival after receiving re-operation and reirradiation, while the clinical benefits disappeared in the subset of IDH-wt. Hence, non-local failure can be regarded as an endpoint event that predicts a poor treatment response.

There are several limitations of this study. Firstly, the fact that it is a retrospective study, means that there has been bias relating to our patient selection. Secondly, given the wide confidence intervals in some subgroups, our results should be interpreted with caution. Additionally, we should continue this study until the last patient reached the endpoint in order to recheck and confirm the results and conclusions in the future. Finally, functional and employment status of LTS in addition to cognition was not recorded which was of great importance in terms of assessing the quality of life (36).

\section{CONCLUSIONS}

Despite improvements of median survival achieved in recent years, the percentage of patients surviving more than five years after diagnosis of GBM remains low. IDH-wt and IDH-mut LTS 
are two distinct subgroups which differ radically in terms of clinical, radiological, and molecular characteristics. Our findings provide a new approach for physicians to better understand the IDH-wt and IDH-mut GBM, which may contribute to developing more tailored therapeutic strategies for patients.

\section{DATA AVAILABILITY STATEMENT}

The original contributions presented in the study are included in the article/Supplementary Material. Further inquiries can be directed to the corresponding author.

\section{ETHICS STATEMENT}

The studies involving human participants were reviewed and approved by Institutional Review Board of Capital Medical University. The patients/participants provided their written informed consent to participate in this study.

\section{AUTHOR CONTRIBUTIONS}

Acquisition of data: HJ, ML, CY, XZ, and QZ. Analysis and interpretation of data: HJ, KY, YC, and XR. Statistical analysis: HJ and KY. Drafting the article: HJ and SL. Funding acquisition: SL and YC. Conception and design: HJ, GZ, and SL. Study supervision: SL. All authors contributed to the article and approved the submitted version.

\section{REFERENCES}

1. Ostrom QT, Cioffi G, Gittleman H, Patil N, Waite K, Kruchko C, et al. Cbtrus Statistical Report: Primary Brain and Other Central Nervous System Tumors Diagnosed in the United States in 2012-2016. Neuro Oncol (2019) 21:v1-v100. doi: 10.1093/neuonc/noz150

2. Stupp R, Mason WP, van den Bent MJ, Weller M, Fisher B, Taphoorn MJ, et al. Radiotherapy Plus Concomitant and Adjuvant Temozolomide for Glioblastoma. N Engl J Med (2005) 352:987-96. doi: 10.1056/NEJMoa043330

3. Stupp R, Taillibert S, Kanner A, Read W, Steinberg D, Lhermitte B, et al. Effect of Tumor-Treating Fields Plus Maintenance Temozolomide vs Maintenance Temozolomide Alone on Survival in Patients With Glioblastoma: A Randomized Clinical Trial. JAMA (2017) 318:2306-16. doi: 10.1001/ jama.2017.18718

4. Marenco-Hillembrand L, Wijesekera O, Suarez-Meade P, Mampre D, Jackson C, Peterson J, et al. Trends in Glioblastoma: Outcomes Over Time and Type of Intervention: A Systematic Evidence Based Analysis. J Neurooncol (2020) 147:297-307. doi: 10.1007/s11060-020-03451-6

5. Cantrell JN, Waddle MR, Rotman M, Peterson JL, Ruiz-Garcia H, Heckman MG, et al. Progress Toward Long-Term Survivors of Glioblastoma. Mayo Clin Proc (2019) 94:1278-86. doi: 10.1016/j.mayocp.2018.11.031

6. Burgenske DM, Yang J, Decker PA, Kollmeyer TM, Kosel ML, Mladek AC, et al. Molecular Profiling of Long-Term IDH-Wildtype Glioblastoma Survivors. Neuro Oncol (2019) 21:1458-69. doi: 10.1093/neuonc/noz129

7. Wong D, Yip S. Finding a Four-Leaf Clover-Identifying Long-Term Survivors in IDH-Wildtype Glioblastoma. Neuro Oncol (2019) 21:1352-3. doi: 10.1093/ neuonc/noz174

8. Cantero D, Rodriguez de Lope A, Moreno de la Presa R, Sepulveda JM, Borras JM, Castresana JS, et al. Molecular Study of Long-Term Survivors of

\section{FUNDING}

This work was supported by the National Natural Science Foundation of China (81771309) and the Capital's Funds for Health Improvement and Research (2020-2-1075).

\section{ACKNOWLEDGMENTS}

The authors appreciate Dr. Hongyan Chen, Dr. Xiaobin Zhao, and Prof. Xuzhu Chen for their help in evaluating radiological features and resection degree, Department of Neuroradiology, Beijing Tiantan Hospital, Capital Medical University.

\section{SUPPLEMENTARY MATERIAL}

The Supplementary Material for this article can be found online at: https://www.frontiersin.org/articles/10.3389/fonc.2021. 632663/full\#supplementary-material

Supplementary Figure 1 | Panel I: Representative images of local recurrence (A-C). Preoperative image showed a lesion in the left frontal lobe, which was totally removed during operation. While 38 months after operation, the tumor recurred at the resection cavity. Panel II: Representative images of non-local recurrence (A-F). Preoperative images showed a lesion in the left temporal lobe, which was totally removed during operation. After 45 months follow-up, a new non-enhanced lesion far from the original resection cavity was found in the corpus callosum.

Supplementary Figure 2 | No significant difference was observed between IDHwt and IDH-mut LTS in the number of patients who received re-operation (A), reirradiation (B), and rechallenge with chemotherapy (C). NA, not applicable.

Glioblastoma by Gene-Targeted Next-Generation Sequencing. J Neuropathol Exp Neurol (2018) 77:710-6. doi: 10.1093/jnen/nly048

9. Hartmann C, Hentschel B, Simon M, Westphal M, Schackert G, Tonn JC, et al. Long-Term Survival in Primary Glioblastoma With Versus Without Isocitrate Dehydrogenase Mutations. Clin Cancer Res (2013) 19:5146-57. doi: 10.1158/1078-0432.CCR-13-0017

10. Louis DN, Perry A, Reifenberger G, von Deimling A, Figarella-Branger D, Cavenee WK, et al. The 2016 World Health Organization Classification of Tumors of the Central Nervous System: A Summary. Acta Neuropathol (2016) 131:803-20. doi: 10.1007/s00401-016-1545-1

11. Ohgaki H, Kleihues P. The Definition of Primary and Secondary Glioblastoma. Clin Cancer Res (2013) 19:764-72. doi: 10.1158/1078-0432.CCR-12-3002

12. Jiang H, Cui Y, Liu X, Ren X, Lin S. Patient-Specific Resection Strategy of Glioblastoma Multiforme: Choice Based on a Preoperative Scoring Scale. Ann Surg Oncol (2017) 24:2006-14. doi: 10.1245/s10434-017-5843-1

13. Jiang H, Yu K, Li M, Cui Y, Ren X, Yang C, et al. Classification of Progression Patterns in Glioblastoma: Analysis of Predictive Factors and Clinical Implications. Front Oncol (2020) 10:590648. doi: 10.3389/fonc.2020.590648

14. Rathore S, Akbari H, Rozycki M, Abdullah KG, Nasrallah MP, Binder ZA, et al. Radiomic MRI Signature Reveals Three Distinct Subtypes of Glioblastoma With Different Clinical and Molecular Characteristics, Offering Prognostic Value Beyond IDH1. Sci Rep (2018) 8:5087. doi: 10.1038/s41598-018-22739-2

15. Kaur G, Bloch O, Jian BJ, Kaur R, Sughrue ME, Aghi MK, et al. A Critical Evaluation of Cystic Features in Primary Glioblastoma as a Prognostic Factor for Survival. J Neurosurg (2011) 115:754-9. doi: 10.3171/2011.5.JNS11128

16. Jiang H, Ren X, Zhang Z, Zeng W, Wang J, Lin S. Polysomy of Chromosomes 1 and 19: An Underestimated Prognostic Factor in Oligodendroglial Tumors. J Neurooncol (2014) 120:131-8. doi: 10.1007/s11060-014-1526-y 
17. Jiang H, Ren X, Cui X, Wang J, Jia W, Zhou Z, et al. 1p/19q Codeletion and IDH1/2 Mutation Identified a Subtype of Anaplastic Oligoastrocytomas With Prognosis as Favorable as Anaplastic Oligodendrogliomas. Neuro Oncol (2013) 15:775-82. doi: 10.1093/neuonc/not027

18. Zhang GB, Cui XL, Sui DL, Ren XH, Zhang Z, Wang ZC, et al. Differential Molecular Genetic Analysis in Glioblastoma Multiforme of Long- and ShortTerm Survivors: A Clinical Study in Chinese Patients. J Neurooncol (2013) 113:251-8. doi: 10.1007/s11060-013-1102-X

19. Jiang H, Ren X, Zhang W, Ma J, Sui D, Jiang Z, et al. A New Prognostic Scoring Scale for Patients With Primary WHO Grade III Gliomas Based on Molecular Predictors. J Neurooncol (2013) 111:367-75. doi: 10.1007/s11060012-1026-x

20. Tejada S, Aldave G, Marigil M, Gallego Perez-Larraya J, Dominguez PD, DiezValle R. Factors Associated With a Higher Rate of Distant Failure After Primary Treatment for Glioblastoma. J Neurooncol (2014) 116:169-75. doi: 10.1007/s11060-013-1279-z

21. Gately L, McLachlan SA, Philip J, Ruben J, Dowling A. Long-Term Survivors of Glioblastoma: A Closer Look. J Neurooncol (2018) 136:155-62. doi: 10.1007/s11060-017-2635-1

22. Gately L, McLachlan SA, Philip J, Rathi V, Dowling A. Molecular Profile of Long-Term Survivors of Glioblastoma: A Scoping Review of the Literature. J Clin Neurosci (2019) 68:1-8. doi: 10.1016/j.jocn.2019.08.017

23. Field KM, Rosenthal MA, Yilmaz M, Tacey M, Drummond K. Comparison Between Poor and Long-Term Survivors With Glioblastoma: Review of an Australian Dataset. Asia Pac J Clin Oncol (2014) 10:153-61. doi: 10.1111/ ajco. 12076

24. Sonoda Y, Kumabe T, Watanabe M, Nakazato Y, Inoue T, Kanamori M, et al. Long-Term Survivors of Glioblastoma: Clinical Features and Molecular Analysis. Acta Neurochir (Wien) (2009) 151:1349-58. doi: 10.1007/s00701009-0387-1

25. Scott JN, Rewcastle NB, Brasher PM, Fulton D, MacKinnon JA, Hamilton M, et al. Which Glioblastoma Multiforme Patient Will Become a Long-Term Survivor? A Population-Based Study. Ann Neurol (1999) 46:183-8. doi: 10.1002/1531-8249(199908)46:2<183::AID-ANA7>3.0.CO;2-7

26. Armocida D, Pesce A, Di Giammarco F, Frati A, Santoro A, Salvati M. Long Term Survival in Patients Suffering From Glio-blastoma Multiforme: A Single-Center Observational Cohort Study. Diagnostics (Basel) (2019) 9:209. doi: 10.3390/diagnostics9040209

27. Krex D, Klink B, Hartmann C, von Deimling A, Pietsch T, Simon M, et al. Long-Term Survival With Glioblastoma Multiforme. Brain (2007) 130:2596606. doi: 10.1093/brain/awm204

28. Barbus S, Tews B, Karra D, Hahn M, Radlwimmer B, Delhomme N, et al. Differential Retinoic Acid Signaling in Tumors of Long- and Short-Term
Glioblastoma Survivors. J Natl Cancer Inst (2011) 103:598-606. doi: 10.1093/ jnci/djr036

29. Smrdel U, Popovic M, Zwitter M, Bostjancic E, Zupan A, Kovac V, et al. LongTerm Survival in Glioblastoma: Methyl Guanine Methyl Transferase (MGMT) Promoter Methylation as Independent Favourable Prognostic Factor. Radiol Oncol (2016) 50:394-401. doi: 10.1515/raon-2015-0041

30. Nakagawa Y, Sasaki H, Ohara K, Ezaki T, Toda M, Ohira T, et al. Clinical and Molecular Prognostic Factors for Long-Term Survival of Patients With Glioblastomas in Single-Institutional Consecutive Cohort. World Neurosurg (2017) 106:165-73. doi: 10.1016/j.wneu.2017.06.126

31. Zou P, Xu H, Chen P, Yan Q, Zhao L, Zhao P, et al. IDH1/IDH2 Mutations Define the Prognosis and Molecular Profiles of Patients With Gliomas: A Meta-Analysis. PloS One (2013) 8:e68782. doi: 10.1371/journal.pone.0068782

32. Hegi ME, Diserens AC, Gorlia T, Hamou MF, de Tribolet N, Weller M, et al. MGMT Gene Silencing and Benefit From Temozolomide in Glioblastoma. N Engl J Med (2005) 352:997-1003. doi: 10.1056/NEJMoa043331

33. Maldaun MV, Suki D, Lang FF, Prabhu S, Shi W, Fuller GN, et al. Cystic Glioblastoma Multiforme: Survival Outcomes in 22 Cases. J Neurosurg (2004) 100:61-7. doi: 10.3171/jns.2004.100.1.0061

34. Utsuki S, Oka H, Suzuki S, Shimizu S, Tanizaki Y, Kondo K, et al. Pathological and Clinical Features of Cystic and Noncystic Glioblastomas. Brain Tumor Pathol (2006) 23:29-34. doi: 10.1007/s10014-006-0195-8

35. Zeng W, Ren X, Cui Y, Jiang H, Zhang X, Lin S. 1q/19p Co-Polysomy Predicts Longer Survival in Patients With Astrocytic Gliomas. Oncotarget (2017) 8:67104-16. doi: 10.18632/oncotarget.17947

36. Weller J, Tzaridis T, Mack F, Steinbach JP, Schlegel U, Hau P, et al. HealthRelated Quality of Life and Neurocognitive Functioning With LomustineTemozolomide Versus Temozolomide in Patients With Newly Diagnosed, MGMT-methylated Glioblastoma (CeTeG/NOA-09): A Randomised, Multicentre, Open-Label, Phase 3 Trial. Lancet Oncol (2019) 20:1444-53. doi: 10.1016/S1470-2045(19)30502-9

Conflict of Interest: The authors declare that the research was conducted in the absence of any commercial or financial relationships that could be construed as a potential conflict of interest.

Copyright (C) 2021 Jiang, Yu, Cui, Ren, Li, Zhang, Yang, Zhao, Zhu and Lin. This is an open-access article distributed under the terms of the Creative Commons Attribution License (CC BY). The use, distribution or reproduction in other forums is permitted, provided the original author(s) and the copyright owner(s) are credited and that the original publication in this journal is cited, in accordance with accepted academic practice. No use, distribution or reproduction is permitted which does not comply with these terms. 